\title{
Antena Compacta para Aplicaciones en Satélites de Reducido Tamaño
}

\author{
Carlos A. Suárez*, Joel Carroll y Gustavo. A. Puerto \\ Universidad Distrital "Francisco José de Caldas", Facultad de Ingeniería, grupo de investigación: \\ Laboratorio de ingeniería de microondas electromagnetismo y radiación (LIMER), Bogotá-Colombia. \\ (e-mail: csuarezf@udistrital.edu.co; jcarrollv@correo.udistrital.edu.co; gapuerto@udistrital.edu.co).
}

* Autor a quien debe ser dirigida la correspondencia.

Recibido Sep. 30, 2015; Aceptado Nov. 25, 2015; Versión final Ene. 17, 2016, Publicado Jun. 2016

\begin{abstract}
Resumen
Este artículo presenta el desarrollo de una antena de parche en anillo circular cortocircuitado con polarización circular a izquierda (LHCP), con mejoramiento de la relación axial mediante el uso de hendiduras (slits) y prolongaciones (stubs). La geometría total presenta dimensiones de $76 \mathrm{~mm} \times 76 \mathrm{~mm} \times$ $1.6 \mathrm{~mm}$, lo cual permite integrarla en una de las caras de un satélite CubeSat $1 \mathrm{U}$. El diseño propuesto muestra un ancho de banda de impedancia del $3.32 \%$ en el rango de frecuencias de $2.37 \mathrm{GHz}$ a $2.45 \mathrm{GHz}$ para un coeficiente de reflexión inferior a $-10 \mathrm{~dB}$ y una ganancia máxima de $6.2 \mathrm{dBi}$ a una frecuencia central de 2.41GHz. Los resultados de las medidas, comparadas con las simulaciones son similares. Sin embargo, se aprecia una pequeña diferencia en las medidas del diagrama de radiación y del coeficiente de reflexión.
\end{abstract}

Palabras clave: antena de anillo circular cortocircuitado; misiones de satélites pequeños; polarización circular

\section{A Compact Antenna for Small Satellite Applications}

\begin{abstract}
The development of a shorted annular ring (SAR) antenna with left hand circular polarization (LHCP), improving the axial ratio by means of slits and stubs is presented in this paper. The whole structure dimensions are $76 \mathrm{~mm} \times 76 \mathrm{~mm} \times 1.6 \mathrm{~mm}$, therefore it can be integrated into one side of a CubeSat $1 \mathrm{U}$ satellite. The proposed design shows an impedance bandwidth of $3.32 \%$ from $2.37 \mathrm{GHz}$ to $2.45 \mathrm{GHz}$ frequency band, with reflection coefficient inferior to $-10 \mathrm{~dB}$ and maximum gain of $6.2 \mathrm{dBi}$ at a central frequency of $2.41 \mathrm{GHz}$. The results of the measurements, compared with simulation results are similar. However, a small difference in the measurements of the radiation diagram and of the reflection coefficient was observed.
\end{abstract}

Keywords: shorted annular ring antenna; small satellite missions; circular polarization. 


\section{INTRODUCCIÓN}

Las misiones satelitales bajo el estándar CubeSat han permitido a instituciones educativas de algunos países incursionar en la exploración del espacio exterior, fortaleciendo de esta manera sus facultades de ingeniería. De igual manera en el sector privado, a empresas ligadas con el área espacial les ha permitido comprobar el funcionamiento de nuevas tecnologías para el espacio, usando para tal fin este tipo de misiones de reducido costo (Sterling, et al., 2012). Es por esta razón que actualmente se han lanzado cerca de 200 misiones CubeSat con diversos objetivos entre los que se encuentran investigaciones atmosféricas, biológicas, predicción de terremotos, determinación de rutas en el ártico, telemetría, estudios solares, etc. De igual manera, las misiones CubeSat son candidatas atractivas para la exploración de asteroides y planetas como Marte (Yu y Zhou, 2014). Por otra parte, este tipo de misiones imponen restricciones en los subsistemas que hacen parte del satélite, teniendo en cuenta las limitaciones en peso, tamaño, consumo de potencia entre otras, los cuales exigen de los diseños alta eficiencia, efectividad y practicidad (Puig Suari, et al., 2001). En este sentido, uno de los sistemas más críticos de este tipo de misiones es tal vez el de las comunicaciones, encargado de enlazar los segmentos terrestre y satelital para poder enviar desde la estación terrena los comandos de control y recibir los datos de telemetría y de carga útil del satélite.

Una parte imprescindible del sistema de comunicaciones es la antena, la cual típicamente funciona en las bandas de VHF/UHF, haciendo uso de dipolos o monopolos entre otros, sin embargo, este rango de frecuencias sólo permite transmisiones a una tasa de bit baja y generalmente las antenas poseen únicamente polarización lineal, aparte que su tamaño supera las dimensiones del CubeSat, por lo que es necesario implementar un sistema de despliegue de antenas para incorporarlas en el satélite. Teniendo en cuenta lo anterior, algunos investigadores han decidido explorar en el diseño de radios y antenas que operen a frecuencias superiores a las bandas de VHF/UHF, particularmente en el rango libre de la banda $\mathrm{S}(2.3 \mathrm{GHz}-$ $2.45 \mathrm{GHz}$ ), siendo la antena de parche, la tecnología normalmente utilizada en este tipo de misiones para operación en esta banda. Cabe resaltar que el uso de la banda $S$ trae como principal desventaja el hecho que al ser una banda libre, este espectro está muy saturado así que es posible tener interferencias con otras redes, sin embargo, en la estación terrena se espera contar con una antena altamente directiva para lograr establecer el enlace apropiado con el satélite, lo cual ayuda en el control de este problema.

En el diseño de antenas de parche, es deseable contar con la posibilidad de controlar en algún grado la ganancia de la antena u otros parámetros del diagrama que la caracterizan. En el caso de parches microstrip simples, el tamaño de la antena es un parámetro que determina el comportamiento electromagnético de la misma, sin embargo mediante el uso de terminales cilíndricos que cortocircuiten el centro de la antena con el plano de masa, es posible mantener constante la frecuencia de resonancia de la antena de tal manera que para una frecuencia de resonancia determinada, un incremento en el diámetro del terminal de corto, permite incrementar el tamaño del parche y por lo tanto su ganancia puede ser mejorada (Volakis, 2007). Una antena que utiliza este tipo de geometría es la antena en anillo circular cortocircuitado (SAR), de la cual se muestra en la Fig. 1 su vista lateral. En esta figura se nota el radio interno $a$, el cual define el orificio circular que se practica en la antena, el radio $b$ que determina el tamaño del parche y el radio de excitación $\rho$ que determina el punto en el cual se ubicará el puerto de la antena.

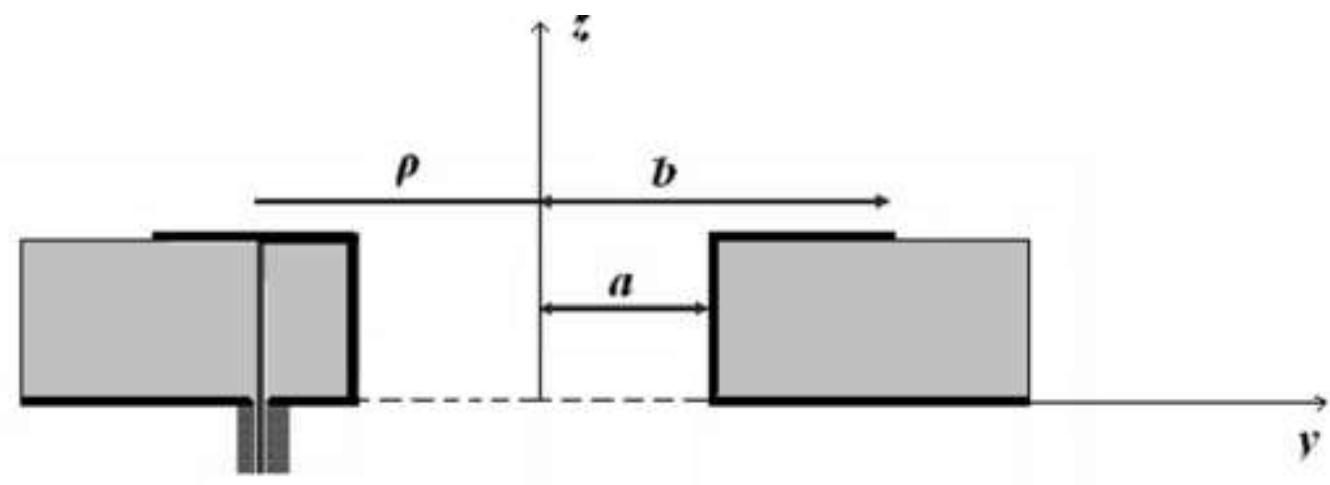

Fig.1: Vista lateral de antena en anillo circular cortocircuitado

En este mismo sentido, en (Jackson, et. al, 1993) se estudia el diseño de una antena de parche en anillo circular cortocircuitado de radio interior $a$, mostrando que la selección apropiada del radio exterior del anillo $b$, permite minimizar la excitación de ondas de superficie, es decir, inhibir la excitación del modo $T M_{0}$ causante de las mismas. De igual manera, en (Basilio L., et. al, 2005) se presenta el diseño de una antena de parche en anillo circular cortocircuitado para aplicaciones en GPS, valiéndose del mismo principio de reducción de las ondas de superficie propuesta en (Jackson, et. al, 1993), mediante la apropiada selección del radio externo del anillo. 
En (Guan et. al, 2015) se propone incrementar la ganancia de una antena con polarización circular mediante el diseño de una agrupación compuesta por cuatro antenas de ranura en anillo circular sobre una guía integrada en sustrato que forma una cavidad logrando una ganancia de $7.1 \mathrm{dBi}$ a $18 \mathrm{GHz}$, un ancho de banda de impedancia de $3.1 \%$ para una magnitud del coeficiente de reflexión de $S_{11}<-10 \mathrm{~dB}$, en el rango de frecuencias de $17.6 \mathrm{GHz}$ a 18.15GHz con un tamaño total de $24 \mathrm{mmX} 24 \mathrm{~mm}$. En (Han et. al, 2015), se presenta el diseño de una antena de parche con polarización y geometría circular, cargada con una línea de transmisión con constante dieléctrica negativa (celdas meta material) mediante una geometría en anillo circular cortocircuitado, logrando una ganancia de $8.5 \mathrm{dBi}$ a una frecuencia de $5.2 \mathrm{GHz}$ sobre un sustrato de tamaño total de $60 \mathrm{~mm} \times 60 \mathrm{~mm}$.

Por otra parte, en (Byun y Choo, 2015) se propone el diseño de una antena de parche con geometría cuadrada y excitación sobre el eje $y$, que incluye un parche parásito acoplado capacitivamente y geometría en anillo cuadrado, el cual es ubicado en la periferia del parche radiante y es separado en dos partes de igual longitud. Mediante los tramos de anillo cuadrado se logra reconfigurar la polarización de la antena de lineal a circular a derechas (RHCP), circular a izquierda (LHCP) o elíptica. La antena hace uso de un sustrato con constante dieléctrica $\varepsilon_{\mathrm{r}}=9$ y una tangente de pérdidas $\tan \delta=0.002$ y el tamaño del plano de masa posee un diámetro de $10 \mathrm{~cm}$, así mismo, la antena presenta en el mejor de los casos una ganancia de $4.9 \mathrm{dBi}$ y un ancho de banda de relación axial de $28 \mathrm{MHz}$ respecto a 3dB. Una estrategia similar es utilizada en (Wu et. al, 2015), donde se adicionan 4 parches parásitos rectangulares ubicados sobre las caras de un parche truncado, los cuales son acoplados capacitivamente para mejorar la relación axial y el ancho de banda de impedancia se incrementa excitando el parche a través de un espacio de aire entre el parche y el plano de masa de $160 \mathrm{~mm}$ de lado, con lo cual se logra un ancho de banda global del 23\% (2.32-2.95 GHz) para una magnitud del coeficiente de reflexión de $S_{11}<-10 \mathrm{~dB}$ y una relación axial inferior a $3 \mathrm{~dB}$.

En (Ryan y Eleftheriades, 2015) se propone el diseño de un parche cuadrado con polarización circular y doble resonancia en las frecuencias de $2.35 \mathrm{GHz}$ y $2.73 \mathrm{GHz}$ y una transparencia del $70 \%$, usando para tal fin un parche de tamaño de $35.75 \times 35.75 \mathrm{~mm}^{2}$ con las esquinas truncadas, impreso sobre un plano de masa de $50 \times 40 \mathrm{~mm}^{2}$. La transparencia se logra cortando agujeros cuadradas de un tamaño de $4.8 X 4.8 \mathrm{~mm}^{2}$ tanto en el parche como en el sustrato formando una malla. La antena incluye bobinas (serpentín) y condensadores impresos sobre el mismo sustrato para controlar la diferencia en fase de los modos ortogonales del parche. Las ganancias máximas obtenidas son de 4.4/4.8dBi y los anchos de banda de impedancia en las dos resonancias para una magnitud del coeficiente de reflexión inferior a $-10 \mathrm{~dB}$ son de $2.33 \mathrm{GHz}-2.42 \mathrm{GHz}$ y de $2.69 \mathrm{GHz}$ a $2.74 \mathrm{GHz}$.

Finalmente, en (Quan et. al, 2014) se presenta el diseño de una antena con polarización circular que hace uso de un parche truncado con geometría en forma de hexágono y un excitador en serpentín. La antena es elaborada sobre 5 capas apiladas de sustrato de $1.57 \mathrm{~mm}$ de espesor, mediante lo cual se obtiene un ancho de banda de impedancia del $42.3 \%$ para pérdidas de retorno de $10 \mathrm{~dB}$ en el rango de $4.24 \mathrm{GHz}$ y $6.76 \mathrm{GHz}$ y un ancho de banda de relación axial del $16.8 \%$ para una referencia de $3 \mathrm{~dB}$, la ganancia máxima obtenida fue de $6.6 \mathrm{dBi}$.

En este artículo se presenta el diseño de una antena con polarización circular a izquierda (LHCP), usando para tal fin una geometría en anillo circular cortocircuitado con el plano de masa (SAR), con lo cual se logra un mejoramiento de la relación axial e incluso de la ganancia, mediante el uso de hendiduras (slits) y prolongaciones (stubs). La geometría propuesta permite que la antena pueda ser fijada a una de las caras de un pico-satélite, ocupando un espacio máximo de $76 \mathrm{~mm} \times 76 \mathrm{~mm} \times 1.6 \mathrm{~mm}$. Los resultados relacionados en este documento muestran que la antena presenta un ancho de banda de impedancia del $3.32 \%$ para una magnitud del coeficiente de reflexión de $S_{11}<-10 \mathrm{~dB}$, en el rango de frecuencias de $2.37 \mathrm{GHz}$ a $2.45 \mathrm{GHz}$ y una ganancia máxima de $6.2 \mathrm{dBi}$ a una frecuencia central de $2.41 \mathrm{GHz}$.

\section{METODOLOGÍA}

El diseño de la antena hace uso de un sustrato con las siguientes especificaciones: espesor $h=1.58 \mathrm{~mm}$, constante dieléctrica de $\varepsilon_{r}=2.33$, tangente de pérdidas Tan $\delta=0.0022$ y mediante el conjunto de ecuaciones (1) a (5) se determinan las dimensiones de cinco parámetros, a saber: el radio interior $a$, el radio exterior $b$, el punto de alimentación ubicado sobre un radio $\rho$ medido desde el centro del parche y ubicado a $135^{\circ}$ respecto al eje horizontal positivo de la antena y el tamaño de las hendiduras y prolongaciones, las cuales hacen parte de la geometría mostrada en la Fig. 2. Esta serie de parámetros determinan el comportamiento electromagnético de la antena en cuanto a ganancia, diagrama, relación axial e impedancia de la antena, partiendo de las restricciones impuestas al tamaño de la antena para ser adosada en una de las caras de un satélite de tamaño $1 U$ (cubo de $100 \mathrm{~mm}$ de lado). 


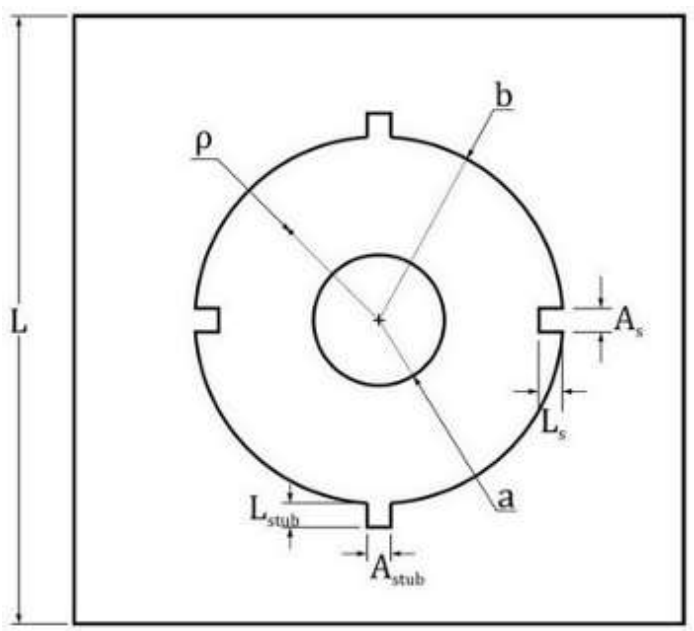

Fig. 2: Vista frontal de la geometría propuesta para la antena.

El tamaño total de la antena y por ende del plano de masa esta determinado por el espacio disponible en una de las caras del satélite, el cual es de $76 \mathrm{~mm} \times 76 \mathrm{~mm} \times 7 \mathrm{~mm}$. Para el diseño de la antena se parte de la ecuación (1), mediante la cual se determina el radio exterior $b$, incluyendo los efectos de borde, aspecto que puede ser tenido en cuenta considerando un radio externo efectivo $b_{e}$ a cambio del radio $b$ y cuya expresión es la obtenida para un parche circular completo (Balanis, 2005).

$$
b=F /\left\{1+\frac{2 h}{\pi \epsilon_{r} F}\left[\ln \left(\frac{\pi F}{2 h}\right)+1.7726\right]\right\}^{1 / 2}
$$

Donde $h$ es el espesor del sustrato, $\varepsilon_{r}$ la constante dieléctrica y $F$ se define en términos de la frecuencia de resonancia $f_{r}$ y la constante dieléctrica cómo:

$$
F=8.791 \times 10^{9} / f_{r} \sqrt{\epsilon_{r}}
$$

Por otra parte, el radio exterior equivalente $b_{e}$ se relaciona en (Balanis, 2005) como en la ec. (3), el cual es necesario para determinar el radio de excitación $\rho$.

$$
b_{e}=b\left\{1+\left(2 h / \pi \epsilon_{r} b\right)[\ln (\pi b / 2 h)+1.7726]\right\}^{1 / 2}
$$

Teniendo en cuenta lo anterior, solucionando la ec. (4) para una impedancia de entrada $R_{\text {in }}(\rho)=50 \Omega$, se determina el radio $\rho$ de alimentación (Balanis, 2005).

$$
R_{\text {in }}(\rho)=\left(1 / G_{t}\right) J_{1}^{2}(k \rho) / J_{1}^{2}\left(k b_{e}\right)
$$

Donde $G_{t}$ es la conductancia total debido a radiación, conducción y dieléctrico y k es el número de onda calculada mediante las expresiones relacionadas en (Balanis, 2005). Por otra parte, la expresión en (5) es la denominada ecuación característica para una cavidad con una pared magnética en $r=b_{e}$, siendo $b_{e}$ el radio exterior efectivo, y una pared eléctrica en $r=a$, teniendo en cuenta que la prima (') indica la derivada de la función de Bessel respectiva (Jackson, et. al, 1993).

$$
J_{n}\left(k_{1} a\right) Y_{n}^{\prime}\left(k_{1} b_{e}\right)=J_{n}^{\prime}\left(k_{1} b_{e}\right) Y_{n}\left(k_{1} a\right)
$$

Partiendo del modelo anterior se diseña una geometría base inicial obteniendo los valores que a continuación se detallan: mediante las ecuaciones (1) - (4) se calcula el radio exterior, resultando $b=34.92 \mathrm{~mm}$ al igual que el radio de excitación con un valor de $\rho=22.9 \mathrm{~mm}$ y con la ec. (5) se calcula el radio interior de $a=16.5 \mathrm{~mm}$. Esta geometría inicial que no incluye las hendiduras ni prolongaciones fue simulada resultando una resonancia cercana a la deseada en la frecuencia de $\mathrm{f}_{0}=2.442 \mathrm{GHz}$, con magnitud del coeficiente de reflexión de $-13.6 \mathrm{~dB}$, sin embargo, los resultados del diagrama de radiación distan de lo deseado dado que se obtiene una ganancia máxima de la antena en las direcciones de referencia (LHCP) y cruzada (RHCP) de solo $\mathrm{G}_{0}=2.19 \mathrm{dBi}$ en ambos casos. Es de anotar que la validez de las anteriores ecuaciones está supeditada al modelo de cavidad utilizado para determinarlas, en el cual se supone que el espesor $h$ del sustrato seleccionado debe ser muy pequeño, condición que normalmente se cumple en los sustratos comerciales en los cuales dicho espesor es típicamente de $h<$ $0.05 \lambda_{0}$. 
Tomando como punto de partida la geometría base inicial antes calculada, la antena se optimizará por intermedio de análisis paramétricos, usando una metodología similar a la propuesta en (Lara, et. al, 2013). Este análisis se lleva a cabo sobre los cinco principales parámetros geométricos de la antena, a saber: el radio interior $a$, el radio exterior $b$, el radio de excitación $\rho$, el largo y ancho de las hendiduras $L_{S}$ y $A_{S}$ y las prolongaciones $L_{s t u b}, A_{s t u b}$ respectívamente. La optimización de la antena se llevó a cabo mediante el código de análisis electromagnético "Ansoft High Frequency Structure Simulator (HFSS)".

La Fig. 3 muestra los diagramas en elevación para las direcciones de referencia (LHCP) y cruzadas (RHCP) para la antena propuesta con hendiduras de dimensiones $L_{S}=4.5 \mathrm{~mm}, A_{S}=2 \mathrm{~mm}$, dos stub con dimensiones de $1.3 \mathrm{~mm}$ de largo y $3 \mathrm{~mm}$ de ancho, los radios interior y exterior de la antena son de $a=13.5 \mathrm{~mm}, \quad b=32 \mathrm{~mm}$ respectivamente. Como se aprecia en la Fig. 3 , la ganancia máxima de la antena en la dirección de referencia (LHCP) es de $G_{0}=6.268 \mathrm{dBi}$ y en la dirección cruzada presenta una ganancia de $G_{0}=-13.71 \mathrm{dBi}$, estableciendo una diferencia entre las dos polarizaciones de $19.978 \mathrm{dBi}$.

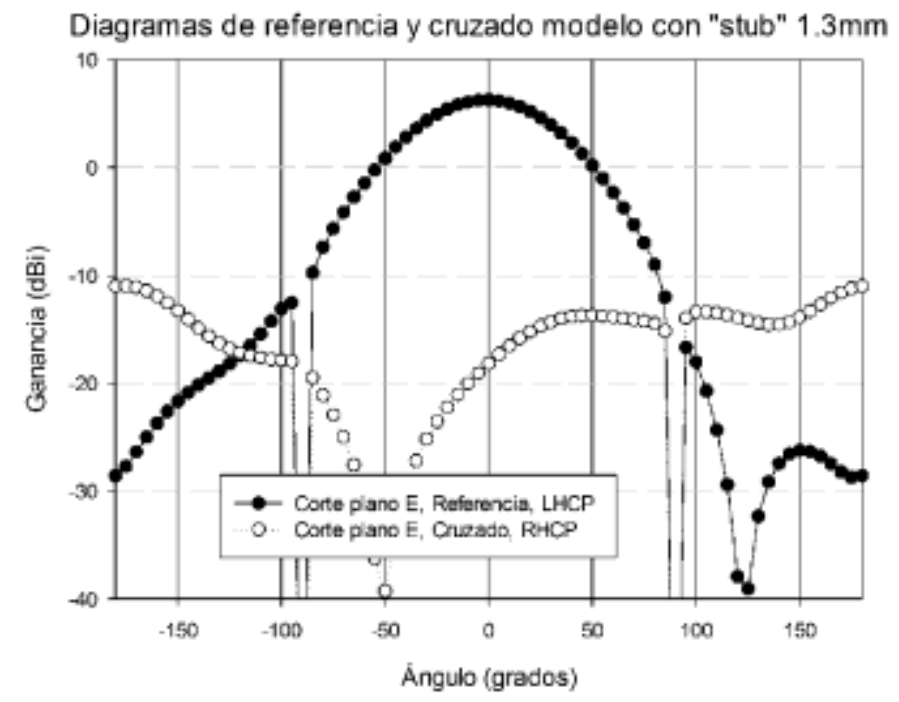

Fig. 3: Diagramas en elevación para las direcciones de referencia y cruzadas para la antena propuesta.

La Fig. 4 muestra el comportamiento de la relación axial de la antena frente al cambio en el tamaño de la prolongación (stub), manteniendo un ancho de $3 \mathrm{~mm}$ en los tres casos. Como se aprecia en la figura, los valores de $1.3 \mathrm{~mm}, 2 \mathrm{~mm}$ y $3 \mathrm{~mm}$ permiten un mejoramiento en este parámetro, particularmente, los valores de $1.3 \mathrm{~mm}$ y $2 \mathrm{~mm}$, arrojan un valor mínimo de $1 \mathrm{~dB}$ de relación axial, siendo mayor el ancho de banda en el caso de $2 \mathrm{~mm}$, sin embargo la selección de uno u otro valor depende de la ganancia obtenida.

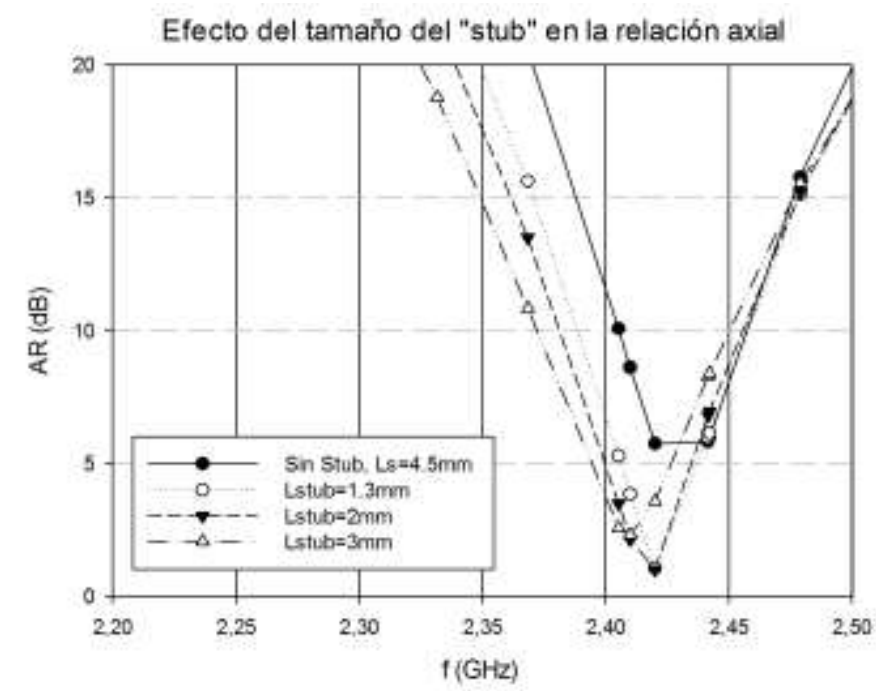

Fig. 4: Diagramas de la relación axial para diversos tamaños de las prolongaciones.

La Fig. 5 muestra el efecto generado sobre la magnitud del coeficiente de reflexión (S11), debido a la ubicación del punto de excitación sobre el círculo de radio $\rho$ (ver Fig. 2). De la Fig. 5 se nota que su incidencia se limita a la adaptación del puerto de la antena a una impedancia de $50 \Omega$ para una frecuencia central de $2.42 \mathrm{GHz}$, la cual es determinada por la relación entre los radios interior y exterior. 


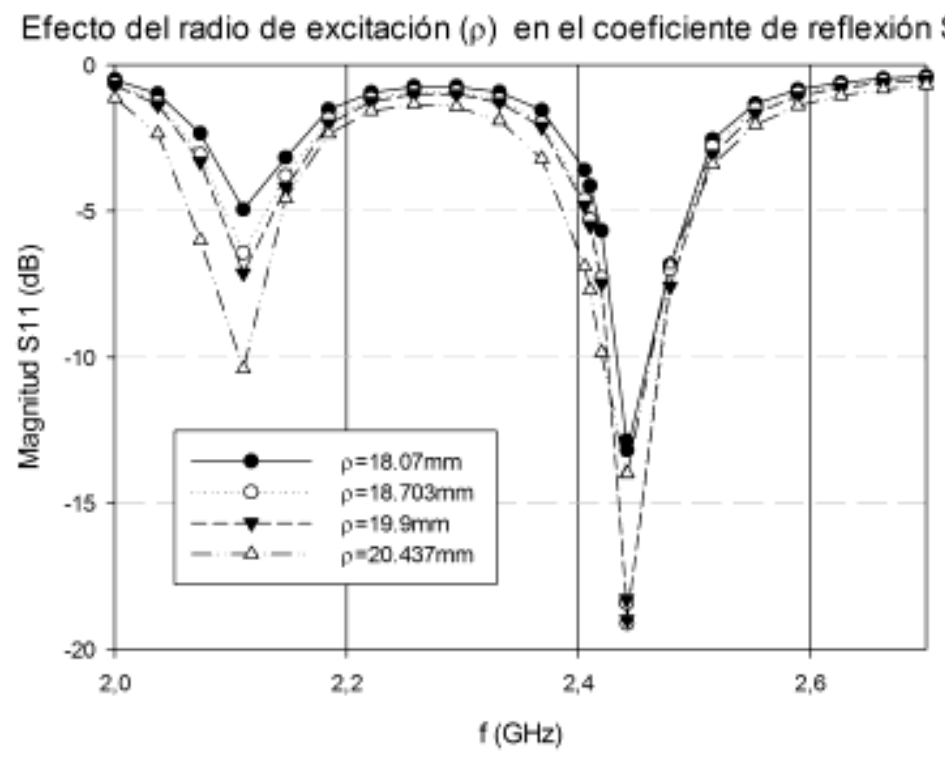

Fig. 5: Efecto del radio de excitación $(\boldsymbol{\rho})$ en el coeficiente de reflexión S11

Como se aprecia en la Fig. 5, este parámetro incide directamente sobre la impedancia de entrada que presenta la antena, de tal manera que la ubicación del punto de excitación en este tipo de geometría es menos critica y ofrece una mayor tolerancia que en el caso de un parche circular completo, teniendo en cuenta que la impedancia de un anillo circular cortocircuitado es del orden de $100 \Omega$ en el borde del anillo, a diferencia de la impedancia de un parche circular completo que presenta impedancia de $0 \Omega$ en el centro del parche a una impedancia en el rango de $200 \Omega$ a $300 \Omega$ en el borde del mismo, de tal manera que la excitación de parche circular completo debe ubicarse en un punto cercano al centro del parche pero con una menor tolerancia.

En la Fig. 5 se aprecia que excitaciones en el rango de $18.703 \mathrm{~mm}$ a $19.9 \mathrm{~mm}$ ofrecen una adaptación de la antena similar y de igual manera esta contribuye a la excitación del modo degenerado sobre una frecuencia de $2.1 \mathrm{GHz}$, siendo más crítico el caso en que la ubicación del punto de excitación esta sobre un radio de $\rho=$ $20.437 \mathrm{~mm}$.

\section{Dimensiones finales de la antena}

Con base en la Fig 2, las dimensiones finales de la antena propuesta como solución se relacionan a continuación: los radios interior y exterior de la antena son de $a=13.5 \mathrm{~mm}, b=32 \mathrm{~mm}$ respectivamente y un radio de excitación del parche de $\rho=18.7 \mathrm{~mm}$, ubicado a $135^{\circ}$ respecto al eje horizontal positivo de la antena, las dimensiones de las hendiduras (slit) son de $L_{S}=4.5 \mathrm{~mm}, A_{S}=2 \mathrm{~mm}$, las dimensiones de las prolongaciones (stub) son de $L_{\text {stub }}=1.3 \mathrm{~mm}, A_{\text {stub }}=3 \mathrm{~mm}$ y el tamaño del plano de masa un cuadrado de lado $L=76 \mathrm{~mm}$ con espesor $h=1.58 \mathrm{~mm}$ sin incluir el conector SMA.

\section{RESULTADOS}

Una vez finalizada la optimización de la geometría de la antena se procedió a fabricar un prototipo por intermedio de una empresa especializada en la elaboración de circuitos impresos, para luego adicionar un conector SMA al puerto de entrada de la antena para llevar a cabo la caracterización de la misma, sin embargo, en el proceso de integración al satélite, se espera conectar directamente el puerto al cable coaxial respectivo y en el otro extremo del cable se suelda el conector SMA que une la antena al radio. El prototipo construido fue caracterizado en impedancia mediante el analizador vectorial de redes de la compañía Rohde\&Schwarz referencia ZVL13 y la caracterización en diagrama fue llevada a cabo en la cámara semi- anecoica disponible en los laboratorios de la Universidad Distrital. La caracterización en impedancia de la antena de igual manera se llevó a cabo dentro de la cámara semi-anecoica para evitar interferencias en el rango de frecuencias de interés. La Fig. 6 muestra la fotografía de la cara frontal de la antena en anillo circular cortocircuitado fabricada.

La Fig. 7 muestra los resultados de las medidas de la magnitud del coeficiente de reflexión $\left(S_{11}\right)$ de la antena, comparado con los resultados obtenidos en las simulaciones en donde se evidencia que los resultados de las medidas presentan un comportamiento similar a los resultados obtenidos en la simulación. También se observa que la antena posee un ancho de banda en el rango de $2.37 \mathrm{GHz}$ a $2.45 \mathrm{GHz}$ para $S_{11}<-10 \mathrm{~dB}$, esto representa un ancho de banda de impedancia porcentual del $3.32 \%$. 


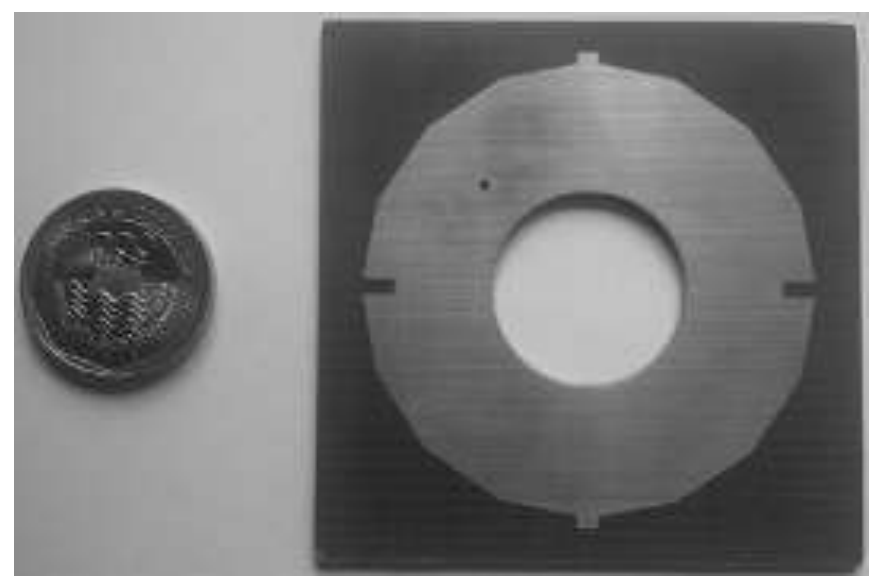

Fig. 6: Fotografía de la vista frontal de la antena de anillo circular cortocircuitada.

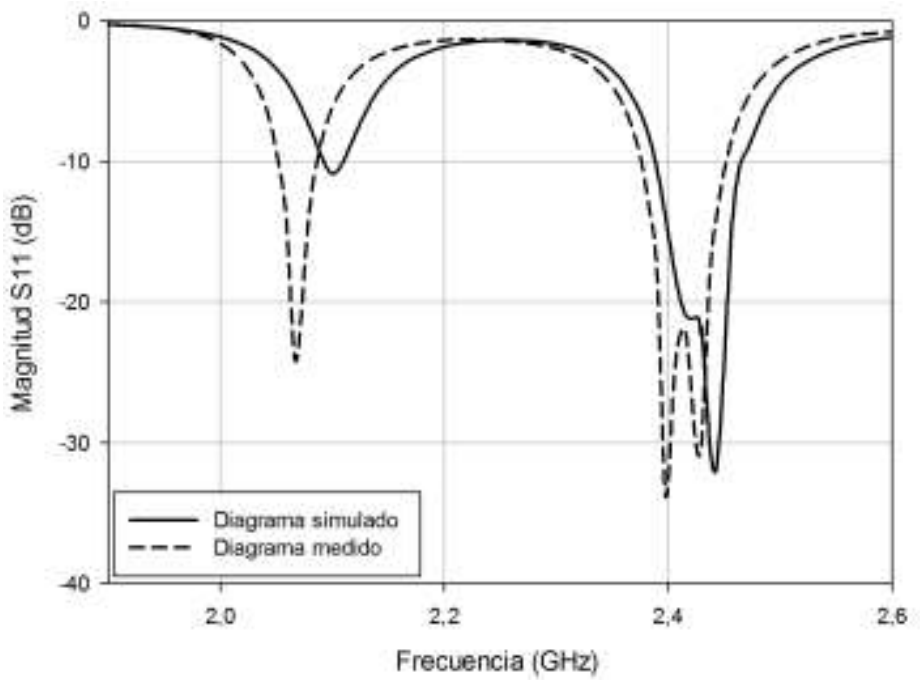

Fig. 7: Resultados de las medidas comparadas con las simulaciones para la magnitud del coeficiente de reflexión ( $\left.S_{11}\right)$.

En cuanto a la ganancia de la antena, en la Fig. 8 se presenta el resultado de las medidas frente a las simulaciones para este parámetro. En la gráfica se observa que al igual que en los otros parámetros, se obtiene un comportamiento similar entre las dos curvas, por otra parte, el nivel máximo de ganancia es de $6.2 d B i$ a la frecuencia de $2.41 \mathrm{GHz}$.

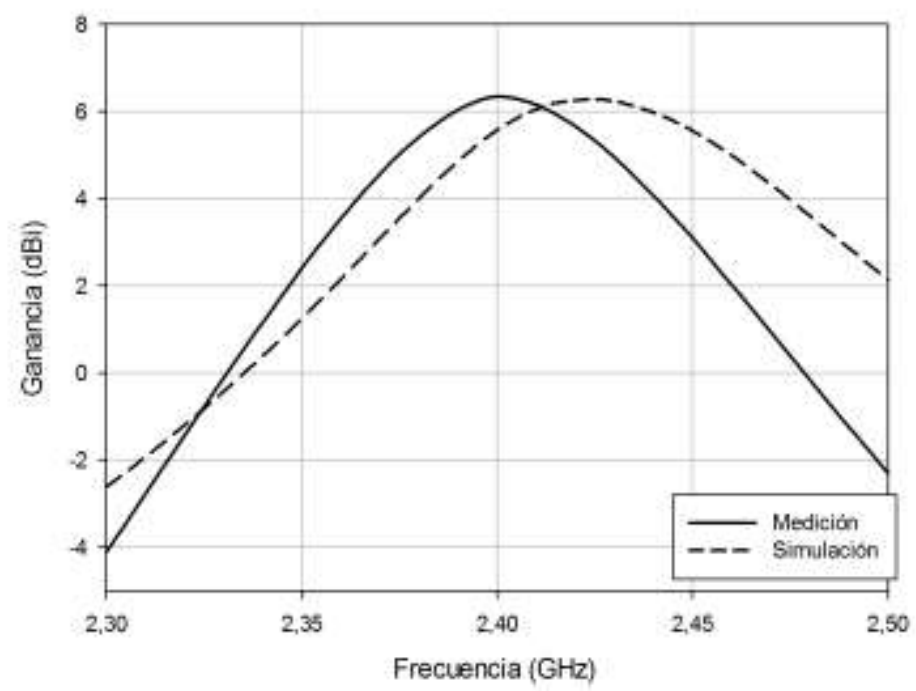

Fig. 8: Resultados de las medidas comparadas con las simulaciones para la ganancia de la antena en la dirección $\theta=0$ y $\phi=0$. 
La Fig. 9 muestra los resultados de las simulaciones comparados con las medidas para los diagramas de radiación en el plano XY a la frecuencia central de $2.41 \mathrm{GHz}$. En esta figura se evidencia que la antena presenta un diagrama de radiación directivo y también se observan diferencias entre los valores esperados en las simulaciones frente a los valores medidos, lo cual se explica por deficiencias en los mecanismos de soporte y cámara anecoica utilizados para este proceso. Finalmente, la Fig. 10 muestra los resultados de las medidas de los diagramas de radiación en las direcciones de referencia y cruzada en el plano $\mathrm{XY}$, para una frecuencia de $2.41 \mathrm{GHz}$.

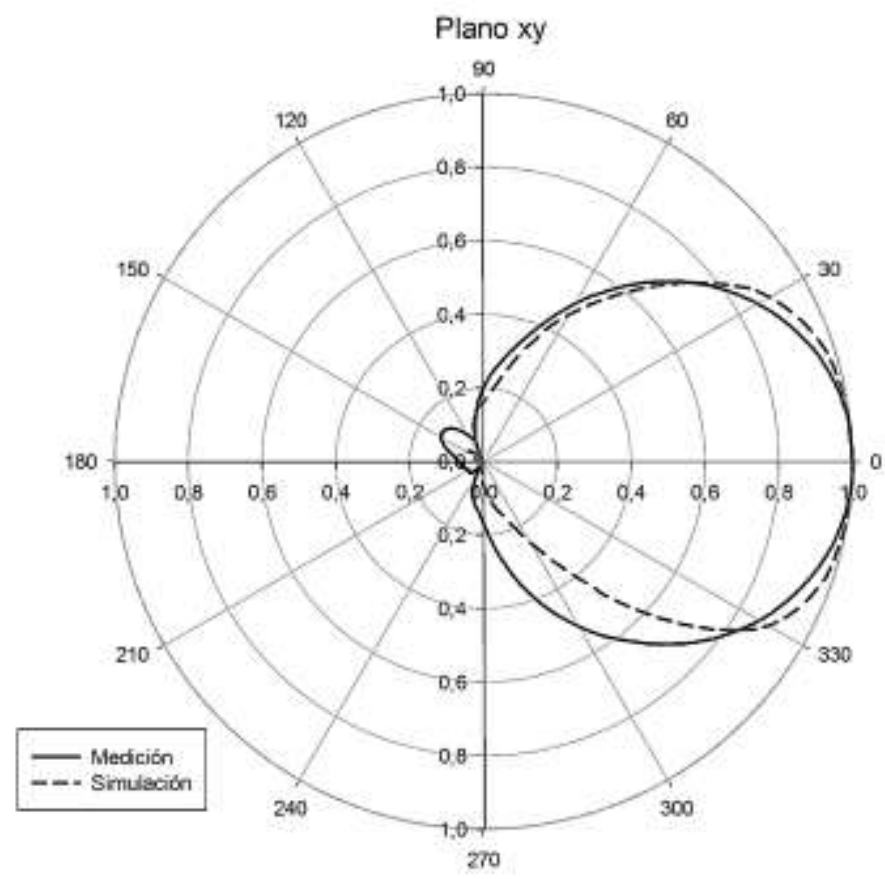

Fig.9: Resultados de las medidas comparados con las simulaciones de los diagramas de radicación normalizados para el corte en el plano XY.

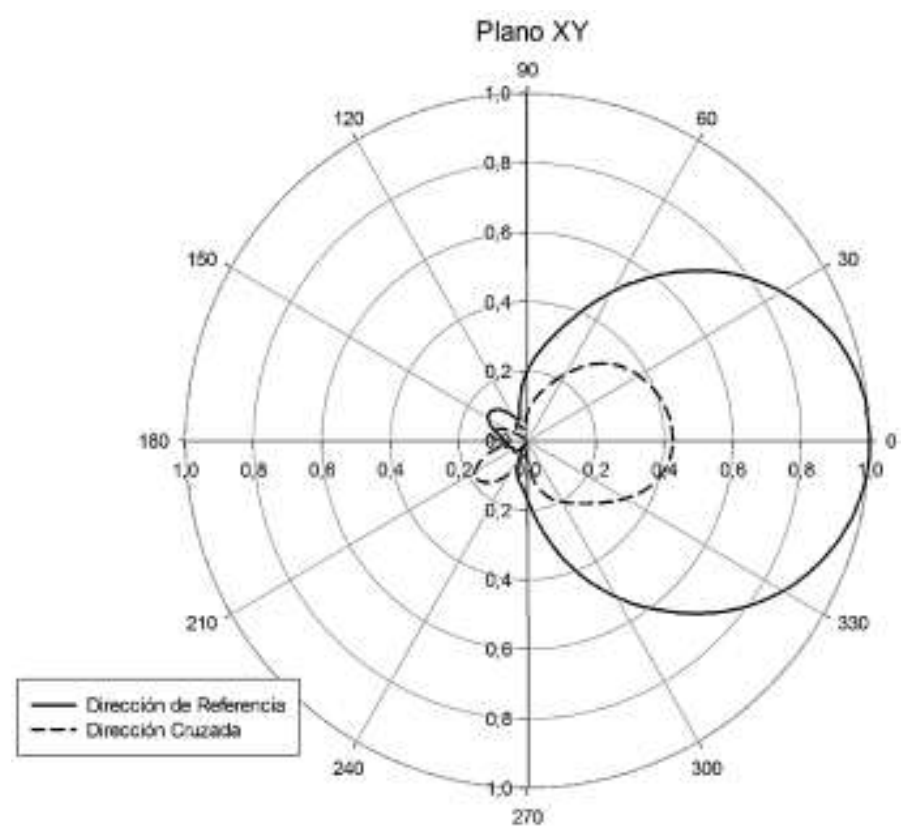

Fig. 10: Resultados de las medidas de los diagramas de radicación de referencia y cruzado en el plano $\mathrm{XY}$.

Esta propuesta frente a las relacionadas en este documento, aparte de permitir ocupar el espacio disponible para el rango de frecuencias deseado y de cumplir con las especificaciones requeridas, posibilita el colocar el lente de una cámara que ocupe el orifico central de la antena aprovechando aun más el reducido espacio disponible en una de las caras de un satélite, sin que por esto se deteriore el comportamiento electromagnético de la antena, así mismo, la combinación apropiada de las dimensiones y ubicación de las hendiduras (slits) y prolongaciones (stubs), permiten optimizar el diagrama de radiación, la ganancia y la relación axial de la antena, sin que esto afecte ostensiblemente la frecuencia central de resonancia de la antena. 


\section{CONCLUSIONES}

En este artículo se presentan los resultados del diseño de una antena con polarización circular a izquierda (LHCP), que hace uso de una geometría en anillo circular cortocircuitado para ser adosada en el espacio disponible $(76 \mathrm{~mm} \times 76 \mathrm{~mm})$ de una de las caras de un satélite CubeSat 1 U. A pesar de las limitaciones de espacio, el diseño propuesto logra una ganancia máxima de $6.2 \mathrm{dBi}$ a la frecuencia central de $2.41 \mathrm{GHz}$ y la relación axial obtenida fue de $0.93 \mathrm{~dB}$ a la misma frecuencia. Así mismo, los resultados de las medidas del ancho de banda de impedancia son del $3.32 \%$ para una magnitud del coeficiente de reflexión de $S_{11}<$ $-10 \mathrm{~dB}$, en el rango de $2.37 \mathrm{GHz}$ a $2.45 \mathrm{GHz}$.

Por último, los resultados de las medidas comparadas con las simulaciones son similares. Sin embargo, se aprecia una pequeña diferencia en las medidas del diagrama de radiación y del coeficiente de reflexión, atribuidos a las limitaciones de la cámara semi-anecoica disponible para tal fin, al igual que a los errores de fabricación y de medida.

\section{REFERENCIAS}

Arnold S. S., R. Nuzzaci y A. Gordon-Ross, Energy Budgeting for CubeSats with an Integrated FPGA, Actas de la conferencia Aeroespacial IEEE, 1-14, Big sky MT., Estados Unidos, 30 de Marzo (2012)

Balanis C. A., Antenna Theory: Analysis and Design, 3a edicion, 844-846, Wiley Interscience, New York, Estados Unidos (2005)

Basilio, L. I., Williams, J.T., Jackson, D.R. y Khayat, M.A., A comparative study of a new GPS reduced-surfacewave antenna, doi: 10.1109/LAWP.2005.851105, IEEE Antennas and Wireless Propagation Letters, (en línea), 4, 233 - 236 (2005)

Byun, G., Choo, H., Antenna polarization adjustment for microstrip patch antennas using parasitic elements, doi: 10.1049/el.2015.0611, IEEE Electronics Letters, (en línea), 51 (14), 1046-1048 (2015)

Guan, D. F., Zhang, Y. S., Qian, Z. P. y Zhang, J. G., Compact circular polarized SIW array antenna with high gain and conical-beam, doi: 10.1049/el.2015.2899, IET, Electronics Letters, (en línea),51(24), 1962-1964 (2015)

Han, W., Yang, F., Long, R., Zhou, L. e Yan, F., Bandwidth enhancement for single-feed circularly polarised microstripantenna with epsilon-negative transmission line-based annular ring, doi: 10.1049/el.2015.1258, IET, Electronics Letters, (en línea), 51(19), 1475-1476 (2015)

Jackson, D.R., Williams, J.T., Bhattacharyya, A.K., Smith, R.L. Buchheit, S.J. y Long, S.A., Microstrip patch designs that do not excite surface waves, doi: 10.1109/8.244643, IEEE Trans. Ant. \& Prop., (en línea), 41(8), 1026-1037 (1993)

Lara, F., Velásquez, N., Sauceda, D. y Acuña, A., Metodología para el Dimensionamiento y Optimización de un Concentrador Lineal Fresnel, doi: 10.4067/S0718-07642013000100013, Inf. Tecnol. (en línea), 24(1), 115$128(2013)$

Puig-suari, J., C. Turner y W. Ahlgren, Development of the Standard CubeSat Deployer and a CubeSat Class PicoSatellite, Actas de la conferencia Aeroespacial IEEE, 347-353, Big sky MT., Estados Unidos, 10 al 17 de Marzo (2001)

Quan, W. L., Hang, W., Xiu, Y. Z. y Hau, W. L., Printed Meandering probe-

Fed Circularly Polarized Patch Antenna with Wide Bandwidth, doi: 10.1109/LAWP.2014.2314141, IEEE

Antennas and Wireless Propagation Letters, (en línea),13, 409-414 (2014)

Ryan, C. G. M., Eleftheriades, G. V., Single- and Dual-Band Transparent Circularly Polarized Patch Antennas With Metamaterial Loading, doi: 10.1109/LAWP.2014.2368115, IEEE Antennas and Wireless Propagation Letters, (en línea), 14, 470-473 (2015)

Volakis J. L (Editor), Antenna Engineering Handbook, 4a Edicion, 24-28, McGraw Hill, Nueva York, Estados Unidos (2007)

Wu, J., Yin, Y., Wang, Z. y Lian, R., Broadband circularly polarized patch antenna with parasitic strips, doi: 10.1109/LAWP.2014.2373823, IEEE Antennas Wirel. Propag. Lett., (en línea), 14, 559-562 (2015)

Yu X. and J. Zhou, CubeSat: A Candidate for the Asteroid Exploration in the Future, Actas de la conferencia internacional en manipulación, manufactura y medida en la escala nano (3M-NANO), 261-265, Taipei, Taiwan, 21 a 31 de Octubre (2014) 
